\title{
BIST Hardware Synthesis for RTL Data Paths Based on Test Compatibility Classes
}

Nicola Nicolici, Bashir M. Al-Hashimi, Andrew D. Brown, and Alan C. Williams

Nicola Nicolici

Bashir M. Al-Hashimi

Andrew D. Brown

Alan C. Williams

Electronic Systems Design Group

Department of Electronics and Computer Science

University of Southampton

Southampton SO17 1BJ

U.K.

Contact address:

Dr. Bashir M. Al-Hashimi

Electronic Systems Design Group

Department of Electronics and Computer Science

University of Southampton

Southampton SO17 1BJ

U.K.

Tel: +44-23-8059-3249

Fax: +44-23-8059-2901

Email: bmah@ecs.soton.ac.uk

A short and preliminary version of this work is published in:

Proceedings of the Design, Automation and Test in Europe (DATE), 1999, pp. 289-295 


\title{
BIST Hardware Synthesis for RTL Data Paths Based on Test Compatibility Classes
}

\begin{abstract}
New BIST methodology for RTL data paths is presented. The proposed BIST methodology takes advantage of the structural information of RTL data path and reduces the test application time by grouping same-type modules into test compatibility classes (TCCs). During testing, compatible modules share a small number of test pattern generators at the same test time leading to significant reductions in BIST area overhead, performance degradation and test application time. Module output responses from each TCC are checked by comparators leading to substantial reduction in fault-escape probability. Only a single signature analysis register is required to compress the responses of each TCC which leads to high reductions in volume of output data and overall test application time (the sum of test application time and shifting time required to shift out test responses). This paper shows how the proposed TCC grouping methodology is a general case of the traditional BIST embedding methodology for RTL data paths with both uniform and variable bit width. A new BIST hardware synthesis algorithm employs efficient tabu search-based testable design space exploration which combines the accuracy of incremental test scheduling algorithms and the exploration speed of test scheduling algorithms based on fixed test resource allocation. To illustrate TCC grouping methodology efficiency, various benchmark and complex hypothetical data paths have been evaluated and significant improvements over BIST embedding methodology are achieved.
\end{abstract}




\section{Introduction}

Register transfer-level (RTL) is the abstraction level in the behavioral domain of the very large scale integration (VLSI) design flow where an integrated circuit is seen as sequential logic consisting of registers and functional units that compute the next state given the current memory state. The functional units which compute the next state logic are arithmetic logic units (ALU), multipliers or complex multi-functional library modules. The complexity of modern digital circuits requires automated synthesis and optimization techniques that can explore a wide class of implementations choices using computer-aided design (CAD) tools [1]. High level-synthesis is the process of generating RTL structure from a behavioral description [2]. The modules (functional units) allocated by high level synthesis algorithms are generated by module generators which are able to synthesize the layout of modules with high performance and device density. The modules are placed in module libraries and have identical physical information. Given the complexity of modern digital circuits it is necessary that testability is addressed at RTL due to fewer elements than at the gate level which makes test synthesis and test scheduling problems more tractable.

\subsection{Previous work}

Two main approaches have been proposed to enhance the testability of digital circuits at RTL. The first approach is aimed at minimizing the complexity of automatic test pattern generation (ATPG). In [3] the complexity of ATPG for scan-based design for testability (DFT) techniques is reduced by an efficient selection of scan flip-flops using RTL information. The high test application time associated with scan-based technique is overcome by using scan chain reconfiguration to reduce shifting time [4] and use of partial scan design of RTL circuits [5]. However, a significant disadvantage of the scan-based technique is that at-speed testing with the complete test set is not possible (i.e., all test patterns cannot been applied at the operational speed of the circuit). To solve at-speed testability, nonscan DFT techniques applicable to RTL data paths were proposed in [6]. Instead of selecting flip-flops to make controllable/observable as the conventional scan-based techniques, execution units are selected using an execution unit graph. Performing at-speed testability and reducing test area overhead is achieved at the cost of expensive test pattern generation phase. To reduce the costs of test pattern generation, an algorithm that adds minimal test hardware in order to ensure that all the embedded modules in the circuit are hierarchically testable was presented in [7]. In [8] a technique for extracting functional (control/data flow) information from RTL controller/data path is presented, thus avoiding the 
use of high level information [7]. Recently in [9] a testability analysis methodology for modular designs is introduced which extracts a set of justification and propagation requirements based on the cone-of-logic of each input and output. However, despite reducing both area overhead and ATPG complexity the test application time and the volume of output data are still high.

The second approach to enhance testability of RTL circuits is built-in self-test (BIST) [10]. While scan BIST [11] eliminates the use of ATPG, it still requires high test application time and volume of output data associated with scan based design. On the other hand parallel BIST reduces both test application time and volume of output data [10]. From now onwards BIST hardware synthesis refers to parallel BIST test hardware insertion for RTL data paths. BIST hardware synthesis at RTL can be further subdivided to functional-based and structural-based BIST hardware synthesis. Functional-based BIST hardware synthesis based on algorithmic and deterministic BIST scheme was presented in [12].This algorithm uses a high-level cell fault model, and data paths are assumed to be composed of only specific adders/subtracters and multipliers. Combination of different BIST schemes and reusing pre-existing modules of the data path for functional-based BIST hardware synthesis under heterogenous test schemes was proposed in [13]. Another functional-based BIST hardware synthesis [14] uses the controller netlist to extract the test control/data flow to derive a set of symbolic justification and propagation paths. In [15] regular expression based high level symbolic testability analysis further reduces test area overhead under delay constraints by carefully carefully selecting a small subset of registers to serve as test pattern generators and output response analyzers. Recently, redundancy identification and testability improvement of digital filter data paths was proposed in [16] which restricts to circuits which are described as a network of shift, add, delay, sign-extension and truncation elements. All the previous functional-based BIST hardware synthesis techniques [12-16] depend strongly on the functional information of data path modules and/or high level control/data flow. On the other hand, structural-based BIST hardware synthesis inserts test registers by analysing interconnections between registers and modules in a given RTL netlist, without using the functional information of data path modules or high level control/data flow. This makes structural-based BIST hardware synthesis more suitable at RTL than functionalbased BIST hardware synthesis when only the structural interconnection of data path modules and registers is given. An early structural-based BIST hardware synthesis algorithm at RTL was presented in [17] without taking into account the test application time. Another structuralbased BIST hardware synthesis algorithm that minimizes test application time and BIST area overhead was proposed in [18]. The algorithm, however, has an inefficient testable design space exploration due to fixed test resource allocation, which means that the test hardware is allocated 
before the test scheduling process. Furthermore, the optimization algorithm limits the number of test plans to only four per module, leading to limited number of explored testable designs. To overcome the fixed test resource allocation, simultaneous test hardware insertion and test scheduling was proposed in [19]. While previous test scheduling algorithms [20-22] assumed fixed test resource allocation, the work in [19] presented an incremental test scheduling procedure which overcomes the limited testable design space exploration encountered with fixed test resources. Despite its good performance, the algorithm in [19] is not capable of dealing in low computational time with complex designs such as 32-point discrete cosine transform (DCT), since a branch and bound-based algorithm is employed to explore the testable design space. A recent approach which explores the testable design space during high level synthesis has been proposed in [23]. However, same test length is considered for all data path modules which leads to unnecessary long test application time.

\subsection{Motivation and objectives}

Up to this point, the described structural-based BIST hardware synthesis algorithms have assumed the BIST embedding methodology where every module port is embedded between a test pattern generator and a signature analysis register. This methodology is inefficient due to the following four problems:

a. to achieve low test application time high number of test registers is required which leads to large BIST area overhead and performance degradation.

b. since every module belongs to a different BIST embedding the aliasing can occur for every module tested separately leading to increase in fault escape probability for the entire data path.

c. the increased number of signature analysis registers yields large volume of output data and increases the overall test application time due to the time required to shift out of test responses.

d. the huge size of the testable design space where test synthesis and test scheduling are strictly interrelated leads to long computational time for efficient testable design space exploration.

To overcome the large number of test registers in the BIST embedding methodology (problem a), a methodology based on chaining modules into test paths was described in [24,25]. Randomness and transparency of data path modules [26] are used to guide the simultaneous 
test path generation and test scheduling. Despite reducing the performance degradation, the great number of test patterns for each test path, which are no longer truly pseudorandom, increased the test application time. The test path generation algorithm lacked the global view of the design space and the suboptimal solution depends on the order in which the modules are processed. Furthermore, the pipelined test scheduling for multiple clock cycles test paths increases the complexity of the BIST controller as the design complexity is enlarged. Concurrent checkers $[27,28]$ have been used for reducing fault escape probability (problem b) during offline self-test. While large BIST area overhead solutions based on duplicate circuitry realized in complementary form are described in [27], the results presented in [28] show that extra test hardware required to achieve low fault-escape probability, if designed as a combination of a concurrent checker and signature analysis registers, is more cost-effective than the design using only signature analysis registers. Recently a different approach which combines mutual and signature testing schemes [29] has been proposed for reducing fault escape probability. This approach uses test registers that combine equality comparators and signature analysis registers leading also to reduction in the volume of output data (problem c). However, due to large number of test registers when maximum test concurrency is targeted the problem of BIST area overhead and performance degradation are not solved. The previous approaches [24-29] proposed separate solutions for solving only one of the problems (a) - (c) at the expense of the other problems of the BIST embedding methodology. Furthermore, the interrelation between test synthesis and test scheduling which leads to huge size of the testable design space (problem d) was not solved efficiently by the previously described approaches [17-22] which trade-off the quality of the final solution and computational time.

The aim of this paper is to introduce a new BIST methodology for RTL data paths using a new concept called test compatibility classes which reduces test application time with comparable or even lower BIST area overhead when compared to the traditional BIST embedding methodology. The proposed BIST methodology which targets data flow intensive application domains, like digital signal processing, communications and graphics, overcomes the performance degradation, fault-escape probability and volume of output data associated with the BIST embedding methodology. Furthermore efficient heuristics for testable design space exploration produce high quality of the final solution in low computational time. The paper is organized as follows. Section 2 introduces the TCC grouping methodology. BIST hardware synthesis for TCC grouping is given in section 3. Experimental results of benchmark and complex hypothetical data paths are presented in section 4. Finally, concluding remarks are given in section 5. 


\section{New BIST methodology for RTL data paths}

This section motivates the key ideas presented in this paper through examples and gives formal concepts and definitions of the proposed BIST methodology. First the shortcomings of the traditional BIST embedding methodology are identified and benefits of the proposed BIST methodology are outlined using a detailed example. Then the formal definition of test compatibility classes is given.

\subsection{An illustrative example}

Traditional BIST embedding methodology embeds every module port between a test pattern generator and a signature analysis register. This may lead to conflicts between different test resources when maximum test concurrency is targeted. Furthermore the number of test resources for low test application is extremely high leading to both high BIST area overhead and performance degradation. The proposed BIST methodology takes advantage of the structural information of RTL data path and reduces the test application time by grouping same-type modules into test compatibility classes (TCCs). Two modules are of the same type if they are two different instances of the same module library prototype and hence they have the identical physical and structural information. Due to the identical physical and structural information the fault sets of two same-type modules have the same detection probability profile [30]. Thus, the same test pattern generators can be used simultaneously (no need to schedule the tests at different test times) for two or more same-type modules without decreasing the fault coverage. On the other hand fault sets of different-type modules have different detection probability profiles and hence different test pattern generators and different test application times are needed to satisfy the required fault coverage. It should be noted that use of hard macro implementations of library modules which have identical physical and structural information can significantly improve the final design [31]. Furthermore design methodologies which use regular elements and identify similarity need to be incorporated in state of the art CAD tools [31,32]. Therefore the proposed BIST methodology is targeting design flows that use few pre-designed module types with identical physical and structural information and exploits the regularity of the data path to reduce test application time and BIST area overhead as explained in the following example.

Example 1 To give insight of the proposed BIST methodology consider the simple data path shown in Figure 1(a). The data path has 3 modules of module-type $A_{\text {type }}$ and 9 registers. Each module is annotated with its name $\left(A_{1}-A_{3}\right)$. In order to test all the modules in a single test session, all the registers are modified into linear feedback shift registers (LFSRs) and multiple- 
input signature registers (MISRs). Each test pattern generator $\left(L F S R_{1}-L F S R_{6}\right)$ applies test patterns to each input port of every module and each signature analysis register $\left(M_{I S R_{7}}-M_{I S R_{9}}\right)$ evaluates the output responses of every module. The paths from test pattern generators to module input ports through multiplexers are denoted by dotted lines. In order to test all the modules simultaneously 6 LFSRs and 3 MISRs are required. Note the number of 2-to-1 multiplexers in the data path is 4 and hence there are $2^{4}$ paths to apply test patterns to module input ports. Any other configuration of test registers which implies sharing of a test resource in order to reduce BIST area overhead will lead to an increased number of test sessions and hence test application time. However, if $A_{\text {type }}$ modules are instances of the same module library prototype, then they have identical physical and structural information and hence identical detection probability profile. Thus if test patterns are applied to modules $A_{1}-A_{3}$ simultaneously same fault coverage is achieved when compared to applying test patterns in different test sessions. Note that $L F S R_{1}$ is the only test register which applies test patterns to left input port of module $A_{1}$ and is connected to left input ports of $A_{2}$ and $A_{3}$. If multiplexers at left input ports of $A_{2}$ and $A_{3}$ select $L F S R_{1}$ as shown in Figure 1(b), the test registers $L F S R_{3}$ and $L F S R_{5}$ (Figure 1(a)) are unnecessary. This clearly leads to savings both in BIST area overhead and performance degradation, since registers $R_{3}$ and $R_{5}$ (Figure $1(\mathrm{~b})$ ) are not modified to perform test functions. Similarly if multiplexers at right input ports of $A_{1}$ and $A_{2}$ select $L F S R_{6}$ (Figure 1(b)), the test registers $L F S R_{3}$ and $L F S R_{5}$ are unnecessary. When same test patterns are generated by $L F S R_{1}$ and $L F S R_{6}$ at input ports of $A_{1}, A_{2}$, and $A_{3}$ same output responses are expected at the same time. Hence a comparator $C A$ is used to check the output responses. Only a single signature analysis register $\mathrm{MISR}_{7}$ is allocated to compress the output responses of all the modules $A_{1}, A_{2}$, and $A_{3}$ which are tested simultaneously. The signature analysis register $\mathrm{MISR}_{7}$ is necessary to detect faults in the case when output responses of all the three modules $A_{1}, A_{2}$, and $A_{3}$ are equal during the entire test application period but different from the fault-free output response. The use of comparators to check the output responses of all the same-type modules tested concurrently solves three problems. Firstly it reduces both BIST area overhead (1 MISR and 1 comparator vs. 3 MISRs) and performance degradation (1 MISR vs. 3 MISRs embedded in data path). Secondly it reduces fault-escape probability since faulty output responses which map into fault-free signatures in the BIST embedding methodology will be detected by the comparators. And thirdly, the number of signatures is reduced which has the following two implications. On one hand the volume of output data is reduced which leads to less storage required for test data. On the other hand the overall test application time is reduced due to less clock cycles needed to shift out the test responses. For example, given the data path width has 8 bits width, the time required to shift 
out the output response stored in $M I S R_{7}, M I S R_{8}$, and $M I S R_{9}$ (Figure 1(a)) is 24 clock cycles when compared to only 8 clock cycles required to shift out the output response stored in $\mathrm{MISR}_{7}$ (Figure 1(b)).

Solutions using comparators described in [27] to enhance fault escape probability are based on duplicate circuitry realized in complementary form leading to huge BIST area overhead. The proposed BIST methodology is fundamentally different where no duplicate circuitry is required and comparators are checking the responses of same-type modules which are instances of same module prototype. This makes the proposed BIST methodology suitable for complex data paths with high number of same-type modules generated automatically by modern CAD tools. The goal of the proposed BIST methodology is to test all the modules of the data path which are random pattern resistant and present testability problems. A part of the steering logic and interconnections are tested for free while testing the modules. It is known that a set of four vectors is sufficient to test a 2-to-1 multiplexer of any bit width. Similarly the functional registers are C-testable and non random pattern resistant structures. The comparators are the only extra DFT hardware added for BIST purposes. Approximately $2 \times n \times k$ test patterns are required to test a $n$-input $k$-bit comparator. Any portion of the data path not tested by the proposed BIST methodology is tested using a small global set of functional patterns. Since comparators check the responses of same-type modules which are inherently different cones-of-logic the small global test of functional patterns can be generated easily using the justification/propagation techniques $[8,9]$. The small global set of functional patterns is applied in a preliminary phase and has no impact on the overall test application time.

\subsection{Definition of Test Compatibility Classes}

An RTL data path consists of $n_{\text {reg }}$ registers, $n_{\text {mod }}$ two-input modules of $n_{\text {res }}$ module-types, and multiplexers. Before test compatibility class concept is introduced, it is necessary to present the following preliminary definitions.

Definition 1 A test register $R_{x}$ performs the test pattern generation function (TPGF) for input port $k\left(I P_{k}\right)$ of module $M_{a}$ if test patterns for $I P_{k}$ of $M_{a}$ are provided by $R_{x}$. Let $I R S\left(M_{a}, I P_{k}\right)$ denote the set of registers that are connected to $I P_{k}$ of $M_{a}$ through only multiplexers. One and only one test register from $I R S\left(M_{a}, I P_{k}\right)$ is performing TPGF for $I P_{k}$ of $M_{a}$.

The test registers used to perform TPGF are: LFSRs, built-in logic block observers (BILBOs) and concurrent BILBOs (CBILBOs). If for each input port $l\left(I P_{l}\right)$ of every data path module, $l=1 \ldots 2 \times n_{\text {mod }}$, there is an $m_{l}$-to- 1 multiplexer then the total number of paths to drive test 
patterns to data path modules is $\prod_{l=1}^{2 \times n_{\text {mod }}} m_{l}$. The testable data paths where both input ports of a module receive same test patterns are not valid in the proposed BIST methodology due to the correlation between identical test patterns at both input ports which leads to a substantial decrease in fault coverage.

Definition 2 Two same-type modules, $M_{a}$ and $M_{b}$, are incompatible, i.e. they cannot be tested simultaneously, if there is a test register $R_{x}$ that performs TPGF for input port $1\left(I P_{1}\right)$ of $M_{a}$ and input port $2\left(I P_{2}\right)$ of $M_{b}$, or if there is a test register $R_{y}$ that performs TPGF for $I P_{2}$ of $M_{a}$ and $I P_{1}$ of $M_{b}$. Two different-type modules are incompatible if there is a test register $R_{z}$ that performs TPGF for any input port of $M_{a}$ and any input port of $M_{b}$. Two modules are compatible if they are not incompatible.

Having described test module incompatibility, now the formal definition of test compatibility classes is given.

Definition 3 A test compatibility class $T C C_{i, j}$ ( $i$ is called the class index, while $j$ is called the module-type index) is a set of modules that satisfies the following three properties:

i. all the modules from $T C C_{i, j}$ are compatible and belong to the same module-type $j$

ii. two test compatibility classes, $T C C_{p, j}$ and $T C C_{q, l}$, are incompatible if for at least one module $M_{a}$ from $T C C_{p, j}$ there exists at least one module $M_{b}$ from $T C C_{q, l}$ such that $M_{a}$ and $M_{b}$ are incompatible.

iii. modules from $T C C_{i, j}$ are tested simultaneously by same test patterns and the output responses are checked by an $n$-input $k$-bit comparator, where $n$ is the cardinality of $T C C_{i, j}$ and $k$ is the bit-width of the data path. A single signature analysis register compresses the output response of a single module from $T C C_{i, j}$ to verify that the output response sequence is correct.

The first property of TCCs (Definition 3-(i)) guarantees that all the modules from a TCC can share test pattern generators leading to less BIST area overhead and performance degradation, without any penalty in test efficiency or test application time. The second property of TCCs (Definition 3-(ii)) indicates that high number of incompatible modules should be merged in a small number of incompatible TCCs leading to maximum test concurrency and hence reduction in test application time. The use of comparators described in the third property of TCCs (Definition 3-(iii)) decreases fault escape probability. Furthermore the reduction in the number of 
signature analysis registers leads to smaller volume of output data and overall test application time (the sum of test application time and shifting time required to shift out test responses). Let $\operatorname{ORS}\left(M_{k}\right)$ denote the set of registers that are connected to the output of module $M_{k}$ through multiplexers only. The output register set of $T C C_{i, j}, \operatorname{ORS}\left(T C C_{i, j}\right)$, is the union of output register sets of all the modules from $T C C_{i, j}$. The signature analysis register for $T C C_{i, j}$ is chosen from $\operatorname{ORS}\left(T C C_{i, j}\right)$. The test registers used for signature analysis are MISRs, BILBOs and CBILBOs. The number of highly expensive CBILBOs required for testing the self-loops in the data path is reduced when using the proposed methodology due to the greater number of potential signature analysis registers for each TCC. While the traditional BIST embedding methodology has $n_{\text {mod }}$ signatures, the proposed BIST methodology has only $\sum_{j=1}^{n_{\text {res }}} n_{\text {classes }}(j)$ signatures, where $n_{\text {classes }}(j)$ is the number of classes for module-type $j$. When $n_{\text {mod }}=n_{\text {res }}$ every module is an instance of a different module library prototype and according to Definitions 1-3 the proposed TCC grouping methodology is identical with the traditional BIST embedding methodology for RTL data paths. Hence, the BIST embedding methodology is a particular case of the TCC grouping methodology when each TCC consists of a single module.

The proposed TCC grouping methodology is not limited to only uniform bit width data paths. Same-type modules which belong to a TCC have the same bit width. However different module types can have different bit width which does not imply any change in the methodology. Definitions 1-3 hold for variable bit width data paths. In the variable bit width case TCCs of different bit width are tested using different bit-width for test registers and $n$-input $k$-bit comparators. Moreover, the proposed methodology can handle both several modules chained together without any registers between them and particular cases when logic/RTL synthesis tools transform different instances of the same module type into different implementations by considering them as new module-types with new detection probability profile.

Example 2 To illustrate Definitions 1-3 consider the data path example of Figure 2, where $L F S R_{1}, L F S R_{2}, L F S R_{3}$ and $L F S R_{4}$ test four modules of $A_{\text {type }}$, and $L F S R_{5}, L F S R_{6}$ and $L F S R_{7}$ test three modules of $B_{\text {type }}$. $L F S R_{1}$ generates test patterns for input port 1 (IP 1 ) of $A_{1}$ and $A_{2}$ and for input port $2\left(I P_{2}\right)$ of $A_{3}$. $L F S R_{2}$ generates test patterns for the $I P_{2}$ of $A_{1}$ and $A_{2}$ and for $I P_{1}$ of $A_{4}$. $L F S R_{3}$ generates test patterns for $I P_{1}$ of $A_{3}$ whilst $L F S R_{4}$ provides test patterns for $I P_{2}$ of $A_{4}$. Modules $A_{1}$ and $A_{2}$ belong to $T C C_{0,0}$ (class index is 0 and $A_{\text {type }}$ index is 0 ). Due to incompatibilities between $A_{1}$ and $A_{3}$ and between $A_{2}$ and $A_{4}$, modules $A_{3}$ and $A_{4}$ belong to $T C C_{1,0}$ (class index is 1 ). Similarly, modules $B_{1}, B_{2}$ and $B_{3}$ are all compatible and belong to $T C C_{0,1}$ (class index is 0 and $B_{\text {type }}$ index is 1 ). Given the bit-width of the data path as 8 bits the 
output responses of modules from $T C C_{0,0}$ are compared by a 2-input 8-bit comparator $\left(C A_{1}\right)$. Similarly, the output responses of modules from $T C C_{1,0}$ are compared by another 2-input 8-bit comparator $\left(\mathrm{CA}_{2}\right)$. On the other hand, output responses of modules from $T C C_{0,1}$ are compared by a 3 -input 8 -bit comparator $\left(C B_{1}\right)$. If any error occurs during testing, the Pass/Fail signal will be activated and the testing process will stop. The output register set $\operatorname{ORS}\left(A_{1}\right)$ is $\left\{R_{7}\right\}$, the $\operatorname{ORS}\left(A_{2}\right)$ is $\left\{R_{8}\right\}$, the $\operatorname{ORS}\left(A_{3}\right)$ is $\left\{R_{7}, R_{9}\right\}$ and the $\operatorname{ORS}\left(A_{4}\right)$ is $\left\{R_{10}\right\}$. Hence the $\operatorname{ORS}\left(T C C_{0,0}\right)$ is $\left\{R_{7}, R_{8}\right\}$ and the $\operatorname{ORS}\left(T C C_{1,0}\right)$ is $\left\{R_{7}, R_{9}, R_{10}\right\}$. Similarly, the $\operatorname{ORS}\left(T C C_{0,1}\right)$ is $\left\{R_{11}, R_{12}\right\}$. Any of these two registers $R_{11}$ and $R_{12}$ can be configured as signature analysis register for $T C C_{0,1}$. The procedure that chooses the best signature analysis register is presented in section 3.3. For data path example in Figure 2 the chosen signature analysis register for $T C C_{0,1}$ is $R_{12}$ whilst both $T C C_{0,0}$ and $T C C_{1,0}$ use $R_{7}$ as signature analysis register at different test times.

\section{New BIST hardware synthesis algorithm for TCC group- ing}

Having described the TCC grouping methodology, now a BIST hardware synthesis algorithm is considered. As outlined in section 2, the BIST embedding methodology is a particular case of the TCC grouping methodology where each TCC consists of a single module. Therefore, testable design space for the TCC grouping methodology is much larger and more complex than testable design space for the BIST embedding methodology. The previous approaches [1722] which trade-off the quality of the final solution and the computational time are unsuitable for the size and complexity of the TCC grouping methodology. This section presents a new and efficient testable design space exploration which combines the accuracy of incremental test scheduling algorithms [19] with the exploration speed of test scheduling algorithms based on fixed test resource allocation [20-22]. Section 3.1 outlines the general framework of tabu search-based testable design space exploration. Section 3.2 presents the generation of new solutions and speed up techniques for local neighborhood search. Finally in section 3.3 an incremental TCC scheduling algorithm for each solution is proposed.

\subsection{Tabu search-based testable design space exploration}

Tabu search [33] was proposed as a general combinatorial optimization technique. Tabu search falls under the larger category of move-based heuristics which iteratively construct new candidate solutions based on the neighborhood that is defined over the set of feasible solutions and the history of optimization. The neighborhood is implicitly defined by a move that specifies 
how one solution is transformed into another solution in a single step. The philosophy of tabu search is to derive and exploit a collection of principles of intelligent problem solving. Tabu search controls uphill moves and stimulates convergence toward global optima by maintaining a tabu list of its $r$ most recent moves, where $r$ is called tabu tenure and it is a prescribed constant. Occasionally, it is useful to override the tabu status of a move when the move is aspirated (i.e., improves the search and does not produce cycling near a local minima). Tabu search based heuristics are simple to describe and implement. Furthermore, a well defined cost function and the use of topological information of the design space will lead to an intelligent search of high quality solutions in very low computational time. Before the proposed tabu search-based testable design space exploration is described, it is necessary to present the following definition.

Definition 4 A solution in the testable design space is a partially testable data path PT-DP where test pattern generators are allocated for each data path module. A fully testable data path FT-DP is generated by allocating signature analysis registers for each test compatibility class of the partially testable data path.

The proposed tabu search-based testable design space exploration is summarized in Figure 3. The algorithm starts with an initial solution which is a partially testable data path PT-DP init $_{\text {it }}$ obtained by randomly assigning a single test pattern generator to each input port of every module from the data path as shown from lines 1 to 4 . During the optimization process (lines 5 to

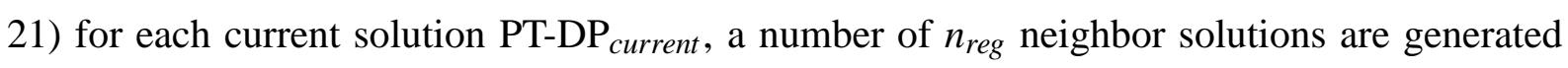
as described in section 3.2. Test application time $T_{x}$ and BIST area overhead $A_{x}$ are computed after a fully testable data path FT-DP $x$ and a test schedule $S_{x}$ are generated using the algorithms from section 3.3, as shown from lines 8 to 12 . The optimization process is guided towards the objective of minimal test application time design by a cost function which is defined as follows.

Definition 5 The cost function is a 2-tuple $C_{x}=\left(T_{x}, A_{x}\right)$, where $T_{x}$ is the test application time, $A_{x}$ is the BIST area overhead and the following relations are defined:

$$
\begin{aligned}
& \text { i. } C_{x 1}=C_{x 2} \text { if }\left(T_{x 1}=T_{x 2}\right) \text { and }\left(A_{x 1}=A_{x 2}\right) \\
& \text { ii. } C_{x 1}<C_{x 2} \text { if }\left(T_{x 1}<T_{x 2}\right) \text { or }\left(T_{x 1}=T_{x 2} \text { and } A_{x 1}<A_{x 2}\right) \\
& \text { iii. } C_{x 1}>C_{x 2} \text { if }\left(T_{x 1}>T_{x 2}\right) \text { or }\left(T_{x 1}=T_{x 2} \text { and } A_{x 1}>A_{x 2}\right)
\end{aligned}
$$

The main objective of the cost function is test application time with BIST area overhead used as tie-breaking mechanism among many possible solutions with same test application time. It 
should be noted that the minimization of other parameters outlined in section 2, performance degradation, volume of output data, overall test application time and fault escape probability, is a by-product of the proposed optimzation using the previously defined cost function. Based on the value of the cost function and on the tabu status of a move, a new solution is accepted or rejected as described from lines 14 to 19 in Figure 3. The tabu list contains registers involved in a move as described in section 3.2. A move is classified as tabu if a register involved in the move is present in the tabu list. The tabu tenure (length of the tabu list) varies from 5 (small designs) to 10 (complex designs). A move is aspirated as shown in line 14 if it has produced a solution which is better than the best solution reached so far. The testable design space exploration continues until the number of iterations since the previous best solution exceeds a predefined $N_{\text {iter }}$.

\subsection{Generation of new solutions and speed up techniques for local neigh- borhood search}

The neighborhood of the current solution in the testable design space PT-DP ${ }_{\text {current }}$ is defined with $n_{\text {reg }}$ feasible neighbor solutions. For each data path register there is a single neighbor solution. Each of the $n_{\text {reg }}$ solutions is provided by an independent subroutine designed to identify better configuration of test registers based on two new metrics. Due to the huge size and complexity of the testable design space, speed up techniques for efficient exploration are required. Before defining the neighbor solution for each register two new metrics and a theorem used for reducing the testable design space are presented.

Definition 6 The current spatial sharing degree $C_{S S D}\left(R_{x}, j, I P_{k}\right)$ of register $R_{x}$ for input port $k\left(I P_{k}\right)$ of module-type $j$ is the number of modules of $j$ for which $R_{x}$ performs test pattern generation function (TPGF) for $I P_{k}$ in the current partially testable data path.

Definition 7 The maximum spatial sharing degree $M_{S S D}\left(R_{x}, j, I P_{k}\right)$ of register $R_{x}$ for input port $k\left(I P_{k}\right)$ of module-type $j$ is the number of modules of $j$ for which $R_{x}$ can perform TPGF for $I P_{k}$. The value of $M_{S S D}\left(R_{x}, j, I P_{k}\right)$ is the cardinality of the set of modules of module-type $j$ whose $I P_{k}$ is connected to $R_{x}$ through only multiplexers.

The following theorem presents a very important theoretical result which has two implications on speeding up local neighborhood search. See [34] for the proof.

Theorem 1 Consider two current solutions, PT-DP ${ }_{\text {current }}^{1}$ and PT-DP ${ }_{\text {current }}^{2}$, with different $C_{S S D}($ $\left.R_{x}, j, I P_{k}\right)$ for given $R_{x}, j$ and $I P_{k}$. In PT-DP ${ }_{\text {current }}^{1}$ the current spatial sharing degree is $0<$ 
$C_{S S D}\left(R_{x}, j, I P_{k}\right)<M_{S S D}\left(R_{x}, j, I P_{k}\right)$, whilst in PT-DP ${ }_{c u r r e n t}^{2}$ the current spatial sharing degree is $C_{S S D}\left(R_{x}, j, I P_{k}\right)=M_{S S D}\left(R_{x}, j, I P_{k}\right)$. Then PT-DP ${ }_{\text {current }}^{2}$ has at most the number of TCCs as PT$\mathrm{DP}_{\text {current }}^{1}$.

The first implication of the theoretical result of Theorem 1 reduces the total testable design space to the representative testable design space. The total testable design space consists of partially testable data paths with all the possible values $0 \leq C_{S S D}\left(R_{x}, j, I P_{k}\right) \leq M_{S S D}\left(R_{x}, j, I P_{k}\right)$ such that all the modules are assigned one and only one test pattern generator. The representative testable design space consists of partially testable data paths for which $C_{S S D}\left(R_{x}, j, I P_{k}\right)$ is considered only $M_{S S D}\left(R_{x}, j, I P_{k}\right)$ such that all the modules are assigned one and only one test pattern generator. Consider the simple data path of Figure 1. In the first case when the current spatial sharing degree for $R_{1}$ is $C_{S S D}\left(R_{1}, A_{\text {type }}, I P_{1}\right)=1$ two more test registers $L F S R_{3}$ and $L F S R_{5}$ are necessary to generate test patterns for $I P_{1}$ of modules $A_{2}$ and $A_{3}$ as shown in Figure 1(a). On the other hand when $C_{S S D}\left(R_{1}, A_{\text {type }}, I P_{1}\right)=M_{S S D}\left(R_{1}, A_{\text {type }}, I P_{1}\right)=3$ only one test pattern generator is necessary to generate test patterns for $I P_{1}$ of all the three modules as shown in Figure 1(b). The case when $C_{S S D}\left(R_{1}, A_{\text {type }}, I P_{1}\right)=1$ has greater BIST area overhead and performance degradation due to $L F S R_{3}$ and $L F S R_{5}$. Furthermore if the simple data path of Figure 1 is a small part of a more complex data path, where $L F S R_{3}$ and $L F S R_{5}$ are already allocated to perform TPGF for different module-types, assigning $L F S R_{3}$ and $L F S R_{5}$ to perform TPGF for $I P_{1}$ of $A_{2}$ and $A_{3}$ respectively, will introduce conflicts between test resources leading to incompatible modules and hence increase in test application time. Theorem 1 justifies the reduction of the total testable design space where all the $C_{S S D}\left(R_{1}, A_{\text {type }}, I P_{1}\right)=\{0,1,2,3\}$ are examined in the search of feasible partially testable data paths to the representative testable design space where only $C_{S S D}\left(R_{1}, A_{\text {type }}, I P_{1}\right)=3$ is considered.

The second implication of the theoretical result of Theorem 1 is concerned with efficient generation of moves in the representative testable design space. Generation of a move in the testable design space for register $R_{x}$ consists of two phases:

i. The first phase computes: $\triangle_{x}\left(j, I P_{k}\right)=M_{S S D}\left(R_{x}, j, I P_{k}\right)-C_{S S D}\left(R_{x}, j, I P_{k}\right) ; \triangle_{x}$ is a metric that measures the difference between the potential and actual use of $R_{x}$ as a test pattern generator for $I P_{k}$ of $j$ modules. Note there are $2 \times n_{\text {res }}$ values of $\triangle_{x}$ for each register $R_{x}$.

ii. In the second phase the move for $R_{x}$ that has the maximum value of $\triangle_{\max }$ is chosen. If there are two or more $j_{m}$ and/or $I P_{k_{n}}$ for which $\triangle_{x}\left(j_{m}, I P_{k_{n}}\right)=\triangle_{\max }$ the move for $j_{m}$ and $I P_{k}$ with the maximum value of $M_{S S D}\left(R_{x}, j_{m}, I P_{k}\right)$ is chosen. 
Let $j_{\max }$ be the index of module-type and $k_{\max }$ be the index of input port for which $\triangle_{\max }$ is maximum. Let $\left\{M_{1}, \ldots, M_{n}\right\}$ be the set of modules of module-type $j_{\max }$ whose $I P_{k_{\max }}$ is connected to $R_{x}$ through only multiplexers. Before the move, $R_{x}$ performs TPGF for $\left\{M_{1}, \ldots, M_{t}\right\}$, whilst $\left\{R_{y_{1}}, R_{y_{2}}, \ldots, R_{y_{m}}\right\}$ perform TPGF for $\left\{M_{t+1}, \ldots, M_{n}\right\}$. After the move, $R_{x}$ performs TPGF for $\left\{M_{1}, \ldots, M_{n}\right\}$, whilst $C_{S S D}\left(R_{y_{i}}, j_{\max }, I P_{k_{\max }}\right)$ are decreased, with $i=1 \ldots m$. The previously described two phases are repeated for each data path register and hence a neighborhood of $n_{\text {reg }}$ feasible solutions is generated. Increasing the current spatial sharing degree of the selected test registers leads to a smaller number of test pattern generators and hence reductions in BIST area overhead and performance degradation. Furthermore, the number of incompatibilities between TCCs is decreased which leads to lower test application time. Moreover the most important feature of the local neighborhood search is the speed up technique for efficient exploration caused by reduction in the size of the testable design space to be explored.

\subsection{Incremental TCC scheduling algorithm}

So far the testable design space to be explored was reduced with respect to the number of test registers required for test pattern generation using the speed up techniques for local neighborhood search. The algorithms outlined in this section further shrink the size of the testable design space by considering simultaneous TCC scheduling and signature analysis registers allocation for each partially testable data path generated by local neighborhood search. Firstly the assignment of every data path module to test compatibility classes to maximize test concurrency is summarized. Secondly the algorithm for simultaneous TCC scheduling and signature analysis registers allocation is described.

To achieve maximum test concurrency it is required that a large number of different-type test compatibility classes are compatible. Following the second property of TCCs (Definition 3-(ii)) a high number of incompatible modules are sought to be merged in a small number of incompatible TCCs. This will reduce the number of edges in the global test incompatibility graph which is defined as follows. A global test incompatibility graph (G-TIG) is a graph where a node appears for every TCC and an edge exists between nodes if test compatibility classes are incompatible. All the edges from G-TIG belong to the edge set E. The generation of G-TIG is carried out in three steps. The first step assigns incompatible same-type modules into test compatibility classes and generates the initial G-TIG. Data path modules that are incompatible with different-type modules are considered in the second step. Unassigned data path modules are assigned to the already existing TCCs such that the number of incompatibilities between different-type TCCs is decreased leading to maximum test concurrency. In the third step unas- 
signed modules which are compatible with all TCCs are added to TCCs such that an increase in the output register set $(O R S)$ of each TCC is achieved. This reduces the number of conflicts between signature analysis registers that are allocated during the test scheduling process leading to lower test application time. The proposed algorithm for generation of G-TIG guarantees by construction that every module is assigned to a TCC and the number of nodes and edges in G-TIG is minimum. This implies maximum test concurrency of the partially testable data path which is a good starting point for the incremental test scheduling algorithm.

Test scheduling is performed using the global test incompatibility graph described previously. Fault sets of different-type modules have different detection probability profiles as outlined in section 2.2. Hence, TCCs of different module-types need different test application times to satisfy the required fault coverage. Thus, the TCC scheduling algorithm deals with unequal test lengths. The test scheduling algorithm for partitioned testing with run to completion from [20] needs to be modified such that test scheduling and signature analysis registers allocation is done simultaneously. Unlike the algorithm $2 \mathrm{M}$ from [20] which schedules tests for a fixed test resource allocation and thus leading to inefficient design space exploration, the proposed algorithm simultaneously schedules TCCs and allocates signature analysis registers. This leads to both more efficient testable design space exploration and higher test concurrency since conflicts between signature analysis registers are avoided. In order to clarify the necessary modifications two more notations are introduced: $U$ is the set of used test registers that have compressed output responses at a previous test time and $B$ is the set of busy test registers that are compressing output responses at the current test time. The following three necessary modifications are carried out to algorithm $2 \mathrm{M}$ from [20] in order to perform simultaneous TCC scheduling and signature analysis register allocation.

i. if all the registers in $\operatorname{ORS}\left(T C C_{i, j}\right)$ are busy at the current test time then test $T C C_{i, j}$ is removed from the candidate node set being postponed for a later test time; otherwise for every available register $R_{k}$ in $O R S\left(T C C_{i, j}\right)$, it is checked whether $R_{k}$ belongs to the used test register set and the $R_{k}$ with the maximum fanin is chosen; this choice will allow $R_{k}$ to be reused at a later test time.

ii. when the shortest currently active test $T C C_{i, j}$ is completed, the test register $R_{k}$ that has served as signature analysis register is removed from the busy register set $B$ and added to the used register set $U$.

iii. after the completion of test scheduling all the registers from the used register set $U$ are modified to signature analysis registers; the algorithm returns a test schedule $\mathrm{S}$ and a fully 
testable data path FT-DP which are used to compute test application time and BIST area overhead in the tabu search testable design space exploration (Figure 3).

The first modification solves the conflicts between signature analysis registers during the test scheduling process reducing both the size of the testable design space to be explored and test application time. Thus the efficiency of testable design space exploration is improved by combining the accuracy of incremental test scheduling algorithms with the exploration speed of test scheduling algorithms based on fixed test resource allocation. The second and third modifications reduce the number of signature analysis registers by reusing them at different test times leading to further reductions in BIST area overhead, performance degradation, and overall test application time.

\section{Experimental results}

The BIST hardware synthesis for the TCC grouping methodology has been implemented on SUN SPARC 20 workstation using 6000 lines of $\mathrm{C}++$ code. To give insight into the efficiency of testability achieved using the presented approach Table 1 shows a comparison of BIST resources and test application time (TAT) using the BIST embedding methodology and the TCC grouping methodology. The results for the BIST embedding methodology were obtained using the same BIST hardware synthesis algorithm assuming that every pair of modules in the data path are different $\left(n_{\text {res }}=n_{\text {mod }}\right)$ as described in section 2.2. The comparison is carried out for a number of benchmark examples including elliptic wave digital filter (EWF) and 8 and 32 point discrete cosine transform (DCT). The benchmarks were synthesized using the ARGEN high-level synthesis system $[35,36]$ for different execution time constraints ranging from 10 to 40. For example, in the case of EWF-17 we have 6 modules (MOD), 3 multipliers (*) and 3 adders (+), and 12 registers (REG). The test application time lengths of adders and multipliers are assumed to be $T_{+}=T$, and respectively $T_{*}=4 \times T$, where $T$ is a reasonably large integer and can be estimated for the required fault coverage using the techniques from [37]. It should be noted that the proposed BIST hardware synthesis algorithm for RTL data paths provides the flexibility of specifying the test application time for the expected fault coverage of $100 \%$ for each data path module. In the experimental results reported in this section it was considered $T=64$, and hence $T_{+}=64$ and $T_{*}=256$, for achieving $100 \%$ fault coverage for each 8 bit data path module which is comparable with the test length values reported in [19]. To validate the assumption regarding test length, adder and multiplier modules have been synthesized and technology mapped into AMS 0.35 micron technology [38]. Subsequently parallel pattern 
single fault propagation fault simulator [39] has shown that $T=64$ is valid for 8 bit data path modules. In general the TCC grouping methodology produces less test registers than the BIST embedding methodology. For example, in the case of EWF-20 the number of LFSRs is reduced from 6 to 4, and the number of MISRs is reduced from 4 to 2. There is further reduction as the design complexity increases. For example, in the case of 32DCT-33 the number of LFSRs is reduced from 30 to 14 , and the number of MISRs is reduced from 19 to 2 . The reduction in test registers in case of TCC grouping is achieved at the expense of comparators. In the case of 32DCT-33 there are one 5 input comparator (C5), one 6 input comparator (C6) and one 8 input comparator (C8). However, the TCC grouping methodology requires reduced BIST area overhead when compared with the BIST embedding methodology as shown in Table 2 for data path width varying from 4-bit up to 16-bit. Note that the proposed BIST methodology is capable of dealing with variable bit width data paths as outlined in section 2.2. BIST area overhead is computed using the equivalent number of 2 input gates required to modify functional registers into LFSRs, MISRs and BILBOs, if applicable, and for implementing the comparators. For benchmark circuit 32DCT-33 the reductions in BIST area overhead in terms of equivalent gates are of $45.63 \%$ in the case of 4-bit data path, $43.72 \%$ in the case of 8-bit data path and $42.65 \%$ in the case of 16-bit data path. But there are cases such as EWF-17 and $8 D C T-13$ where the BIST embedding methodology produces better BIST area overheads. This has been achieved without reaching the minimal TAT. For example circuits EWF-17 and 8DCT-13 reductions of $20 \%$ in TAT are achieved by the TCC grouping methodology. This result is derived using the TAT from Table 1, where the minimum reached TAT for the TCC grouping methodology is $5 \times T$, and for the BIST embedding methodology is $4 \times T$.

So far the reductions in TAT and BIST area overhead achieved by the TCC grouping methodology when compared to the BIST embedding methodology were outlined. Table 2 also shows the reductions in number of test registers (TR), volume of output data (VOD), and overall test application time (overall-TAT). The reduction in TR represents the reduction in the number of data path registers modified in test registers and it may have direct impact on the performance degradation (clock period). For example the reduction in TR for $E W F-17$ is $22.22 \%$. The reduction in TR is increased up to $67.35 \%$ as in the case of 32DCT-33. Similarly the reduction VOD varies from $33.33 \%$ in the case of EWF-17 up to $90.48 \%$ in the case of $32 D C T-30$ and 32DCT-31. The volume of output data is considered directly proportional to the number of signature analysis registers. The number of signature analysis registers is very small due to the large number of modules grouped in TCCs and reuse of signature analysis registers at different test times. The volume of output data does not have impact only on the storage required 
for test data but also on the overall test application time which consists of the test application time (TAT) and the shifting time required to shift out the test responses at the end of the testing process. The shifting time requires $n_{S A} \times k$ clock cycles, where $n_{S A}$ is the number of signature analysis registers and $k$ is the data path width. The last column of Table 2 shows the reduction in overall-TAT given the data path width as 8 bits and $T=64$. It should be noted that due to high test concurrency, low test application time required for each data path module $(T=64)$, and high number of test registers, the value of shifting time is comparable to test application time. This implies that the proposed BIST methodology leads to substantial savings in overall-TAT due to the reduction in signature analysis registers. For all benchmark circuits where both BIST embedding and TCC grouping methodologies achieved minimal test application time $(4 \times T)$ the overall-TAT is reduced in the case of the TCC grouping methodology due to a smaller number of signature analysis registers. For example, in the case 32DCT-30 the overall-TAT reduction achieved by the TCC grouping methodology when compared to the BIST embedding methodology is $35.85 \%$. The BIST hardware synthesis algorithm has excellent computational time. The CPU time required to achieve lowest TAT for benchmark circuits is shown in the last column of Table 1. For example, in the case of EWF and 8 point DCT designs, the computational time varies from $0.7 \mathrm{~s}$ to $3 \mathrm{~s}$. In the case of designs with huge testable design space like 32 point DCT, high quality solutions are achieved in computational times ranging from $38 \mathrm{~s}$ to $130 \mathrm{~s}$. A high quality solution is a fully testable data path with test application time equal (or almost equal) to the longest test application time required to test the most random pattern resistant module $(4 \times T$ in the case of benchmark circuits of Table 1). It should be noted that despite the fact that test registers and test schedule of the final solution are dependent on the initial random assignment of test registers, the quality of the final solution (in terms of test application time and BIST area overhead) is independent on the initial random assignment due to intelligent neighborhood search outlined in section 3 .

Although the emphasis of the work presented in this paper is on built-in self-testable data paths the following discussion clarifies the issue of controlling the controller outputs while testing the data path, and that of testing the controller itself. Since the proposed BIST methodology targets data flow intensive applications there are only a few flip flops required to implement the functional controller. For example, in the case of 32DCT-30 only 5 flip flops implement the 30 control states. Since control signals need to be controlled during testing, a BIST controller is synthesized. However, the size of the BIST controller is small and the BIST controller can easily be merged with the functional controller for both controlling the signals during data path testing and testing the controller itself. This leads to insignificant area overhead when compared 
to the size of the data path. For example, in the case of 32DCT-30 the BIST controller consists of 1 flip flop which indicates that data path is in test mode, 6 flip flops implementing the pattern counter $\left(T=2^{6}\right), 2$ flip flops implementing test application controller $(\mathrm{TAT}=4 \times T)$ and 1 flip flop that switches between the phases of test application and shifting out test responses. Therefore the entire controller (both functional and BIST controller) has only 15 flip flops which is insignificant when compared to the size of data path (60 registers of which 18 LFSR and 2 MISR as shown in Table 1). The control signals controlling the data path are synthesized using both functional and test specification and the control logic (which is insignificant when compared to only one data path multiplier) is tested by configuring controller into a LFSR. This does not add any performance degradation for data flow intensive applications since the clock period is constrained by the critical path in the complex data path. Furthermore, both the size of the merged controller and test application time of the controller is smaller in the case of the proposed TCC grouping when compared to BIST embedding due to smaller number of cycles required to shift out test responses and less logic required to implement control signals during testing. If the random patterns generated by the LFSR are not satisfactory to detect all the faults in the controller some additional DFT such as scan needs to be inserted. To provide the complete test scenario, the work in $[8,9]$ lends itself well for the untested multiplexers and registers since they necessitate only 4 test patterns regardless of the bit width. Therefore it is likely that the size of the global set of functional patterns mentioned in section 2.1 will be small.

The BIST hardware synthesis for TCC grouping methodology allows the huge testable design space to be explored efficiently by combining the accuracy of incremental test scheduling algorithms and the exploration speed of test scheduling algorithms based on fixed test resource allocation, as outlined in section 3. This means it can be used with extremely complex hypothetical designs of dimensions not often reported in literature. Complex hypothetical data paths ${ }^{1}$ have been generated as described in the following. The number of modules $n_{\text {mod }}$ varies from 35 to 45 , and the number of registers $n_{\text {reg }}$ varies from 90 to 115 . The number of module-types is $n_{\text {res }}=5$. The maximum fanin for every register or input port of a module is $M_{\text {fanin }}=8$. The input register set of each input port of every module contains a random number $n_{r}$, with $1 \leq n_{r} \leq M_{\text {fanin }}$, of randomly chosen registers. Similarly, the number of modules multiplexed at the input of each register is a random number $n_{m}$, with $1 \leq n_{m} \leq M_{\text {fanin }}$, of randomly chosen modules. The TAT of three module-types is assumed $T$, and in the case of the other two module-types the TAT is considered $4 \times T$. Figure 4 clearly demonstrates that the TCC grouping methodology overcomes the problems of the BIST embedding in dealing with complex hypo-

\footnotetext{
${ }^{1}$ The complex hypothetical data paths are available on request from the authors
} 
thetical data paths. The complex hypothetical data paths have been labelled $E X-1$ to $E X-9$. For most of the designs the proposed TCC grouping reduces TAT when compared to BIST embedding. However, when both BIST embedding and TCC grouping achieve low TAT, the reductions in BIST area overhead, number of test registers (impact on performance degradation), volume of output data and overall test application time are substantial. For example in the case of EX-9 reductions of $50 \%$ in TAT, $23 \%$ in BAO, $47 \%$ in TR, $94 \%$ in VOD and $61 \%$ in overall TAT are achieved. Furthermore, the computational time for obtaining high quality solutions is still very low related to the size of the testable design space. For example it took less than 600s to find high quality solutions for data paths with 45 modules and up to 115 registers.

Finally, Figure 5 shows how the proposed TCC grouping methodology decreases the faultescape probability when compared to the BIST embedding methodology. The experiments were done for a data path module with possible $10^{6}$ error sequences, where the aliasing error sequences, for a given characteristic polynomial of signature analysis register, vary from 10 to 90. Fault-escape probability of a module varies from $P_{m}=0.01 \%$ to $P_{m}=0.09 \%$. As it can be seen from Figure 5(a), in the case of BIST embedding methodology the fault escape probability for group of modules $\left(P_{g}\right)$ increases as the number of modules tested simultaneously increases. On the other hand, in the case of the TCC grouping, the fault-escape probability decreases exponentially with the number of modules tested simultaneously as shown in Figure 5(b). This is due to the fact that a fault is not detected in the TCC grouping methodology only when initially the $n$-input $k$-bit comparator fails to detect the fault and subsequently the signature of a TCC also fails to detect the fault. A previous work on reducing fault-escape probability at the expense of increased area overhead, performance degradation, and volume of output data was presented in [40]. Note that the proposed methodology does not introduce any area overhead, nor performance degradation, whilst the reduction in fault-escape probability is exponential.

\section{Conclusion}

This paper has addressed the testability of RTL data paths. It has been shown that an improvement in terms of test application time, BIST area overhead, performance degradation, volume of output data, overall test application time (the sum of test application time and shifting time required to shift out test responses) and fault-escape probability is achieved using the newly introduced test compatibility classes-based methodology. The new BIST methodology is based on grouping modules with identical physical information into TCCs and testing the compatible modules by sharing a small number of test pattern generators at the same test time. An $n$-input 
$k$-bit comparator checks module output responses from each TCC reducing the fault-escape probability and the number of signatures that have to be shifted out. The proposed TCC grouping methodology is suitable for RTL data paths with both uniform and variable bit width. A new BIST hardware synthesis uses efficient tabu search-based testable design space exploration which combines the accuracy of incremental test scheduling algorithms with the exploration speed of test scheduling algorithms based on fixed test resource allocation. The huge size of the testable design space is reduced by considering only the representative partially testable data paths during the local neighborhood search. An incremental TCC scheduling algorithm further shrinks the size of the testable design space by generating a fully testable data path using simultaneous test scheduling and signature analysis registers allocation. BIST hardware synthesis algorithm for the proposed TCC grouping methodology has been tested exhaustively for benchmark and complex hypothetical data paths. When compared to the traditional BIST embedding methodology, the TCC grouping methodology is capable of reducing the test application time with comparable of even lower BIST area overhead and high reductions in performance degradation, volume of output data, fault-escape probability and overall test application time. Furthermore the proposed BIST hardware synthesis algorithm achieves high quality of the final solution in low computational time.

The proposed methodology and the BIST hardware synthesis algorithm have been successfully integrated in high-level synthesis design flow [36] leading to lower design cycle by considering testability at higher levels of abstraction than the gate-level. This reinforces the conclusion reached recently by other researchers [6-8] that testability of digital circuits is best explored and optimized at the register transfer level. Since the proposed methodology targets RTL data paths of data flow intensive designs future work will investigate integrated controller/data path testing for both data flow and control flow intensive circuits.

\section{Acknowledgement}

The authors would like to thank Professor Melvin Breuer of University of Southern California for providing a copy of reference [40]. 


\section{References}

[1] G. de Micheli, Synthesis and Optimization of Digital Circuits. McGraw-Hill International Editions, 1994.

[2] M.C. McFarland, A.C. Parker, and R. Camposano, "The high-level synthesis of digital systems," Proceedings of the IEEE, vol. 78, pp. 301-318, Feb 1990.

[3] V. Chickername, J. Lee, and J.K. Patel, "Addressing design for testability at the architectural level," IEEE Transactions CAD, vol. 13, pp. 920-934, Jul 1994.

[4] S. Narayanan and M.A. Breuer, "Reconfiguration techniques for a single scan chain," IEEE Transactions on CAD, vol. 14, pp. 750-765, Jun 1995.

[5] R. Gupta and M.A. Breuer, "Partial scan design of register-transfer level circuits," Journal of Electronic Testing: Theory and Applications (JETTA), vol. 7, pp. 25-46, Aug 1995.

[6] S. Dey and M. Potkonjak, "Nonscan design-for-testability techniques using RT-level design information,” IEEE Transcations on CAD, vol. 16, pp. 1488-1506, Dec 1997.

[7] I. Ghosh, A. Raghunathan, and N.K. Jha, "Design for hierarchical testability of RTL circuits obtained by behavioral synthesis," IEEE Transcations on CAD, vol. 16, pp. 10011014, Sep 1997.

[8] I. Ghosh, A. Raghunathan, and N.K. Jha, "A design for testability technique for RTL circuits using control/data flow extraction," IEEE Transcations on CAD, vol. 17, pp. 706723, Aug 1998.

[9] Y. Makris and A. Orailoglu, "RTL test justification and propagation analysis for modular designs," Journal of Electronic Testing: Theory and Applications (JETTA), vol. 13, pp. 105-120, Oct 1998.

[10] V.D. Agrawal, C.R. Kime, and K.K. Saluja, "A tutorial on built-in self test - part 2: Applications," IEEE Design and Test of Computers, pp. 69-77, Jun 1993.

[11] S. Boubezari, E. Cerny, B. Kaminska, and B. Nadeau-Dostie, "Testability analysis and test-point insertion in RTL VHDL specifications for scan-based BIST," IEEE Transactions on $C A D$, vol. 18, pp. 1327-1340, Sep 1999.

[12] D. Gizopoulos, A. Paschalis, and Y. Zorian, "An effective BIST scheme for datapaths," in Proc. International Test Conference, pp. 76-85, 1996. 
[13] D. Berthelot, M.L. Flottes, and B. Rouzeyre, "BISTing datapaths under heterogenous test schemes," Journal of Electronic Testing: Theory and Application (JETTA), vol. 14, pp. 115-123, Jan 1999.

[14] I. Ghosh, N.K. Jha, and S. Bhawmik, "A BIST scheme for RTL circuits based on symbolic testability analysis," IEEE Transcations on CAD, vol. 19, pp. 111-128, Jan 2000.

[15] S. Ravi, N.K. Jha, and G. Lakshminarayana, "TAO-BIST: A framework for testability analysis and optimization for built-in self-test of RTL circuits," IEEE Transactions on $C A D$, vol. 19, Aug 2000.

[16] L. Goodby and A. Orailoglu, "Redundancy and testability in digital filter datapaths," IEEE Transcations on CAD, vol. 18, pp. 631-644, May 1999.

[17] P.R. Chalsani, S. Bhawmik, A. Acharya, and P. Palchaudhuri, "Design of testable VLSI circuits with minimum area overhead," IEEE Transactions on Computers, vol. 38, pp. 1460-1462, Sep 1989.

[18] A. Basu, T.C. Wilson, D.K. Banerji, and J.C. Majithia, "An approach to minimize testability for BILBO based built-in self-test," in Proc. 5th International Conference on VLSI Design, pp. 354-355, 1992.

[19] S.P. Lin, C.A. Njinda, and M.A. Breuer, "Generating a family of testable designs using the BILBO methodology," Journal of Electronic Testing: Theory and Applications (JETTA), vol. 4, no. 2, pp. 71-89, 1994.

[20] G.L. Craig, C.R. Kime, and K.K. Saluja, "Test scheduling and control for VLSI built-in self-test," IEEE Transactions on Computers, vol. 37, pp. 1099-1109, Sep 1988.

[21] W.B. Jone, C.A. Papachristou, and M. Pereira, "A scheme for overlaying concurrent testing of VLSI circuits," in Proc. 26th Design Automation Conference, pp. 531-536, 1989.

[22] C.I.H. Chen, "Graph partitioning for concurrent test scheduling in VLSI circuits," in Proc. 28th Design Automation Conference, pp. 287-290, 1991.

[23] H.B. Kim, T. Takahashi, and D.S. Ha, "Test session oriented built-in self-testable data path synthesis," in IEEE International Test Conference, pp. 154-163, 1998.

[24] A. Orailoglu and I.G. Harris, "Test path generation and test scheduling for self-testable designs," in Proc. International Conference on Computer Design, pp. 528-531, 1993. 
[25] I.G. Harris and A. Orailoglu, "Microarchitectural synthesis of VLSI designs with high test concurrency," in Proc. 31st Design Automation Conference, pp. 206-211, 1994.

[26] S. Chiu and C.A. Papachristou, "A desing for testability scheme with applications to data path synthesis," in Proc. 28th IEEE/ACM Design Automation Conference, pp. 271-277, 1991.

[27] E.J. McCluskey, "Design for testability," in Logic Design Principles With Emphasis On Testable Semicustom Circuits, pp. 424-488, New Jersey: Prentice Hall, 1986.

[28] S.K. Gupta and D.K. Pradhan, "Utilization of on-line (concurrent) checkers during built-in self-test and vice versa," IEEE Transactions on Computers, vol. 45, pp. 63-73, Jan 1996.

[29] M.F. Abdulla, C.P. Ravikumar, and A. Kumar, "Optimization of mutual and signature testing schemes for highly concurrent systems," Journal of Electronic Testing: Theory and Applications (JETTA), vol. 12, pp. 199-216, June 1998.

[30] P.H. Bardell, W.H. McAnney, and J. Savir, Built-In Self Test - Pseudorandom Techniques. John Wiley \& Sons, 1986.

[31] D.G. Chinnery and K. Keutzer, "Closing the gap between ASIC and custom: An ASIC perspective," in Proc. 37th IEEE/ACM Design Automation Conference, pp. 637-642, 2000.

[32] W.J. Dally and A. Chang, "The role of custom design in ASIC chips," in Proc. 37th IEEE/ACM Design Automation Conference, pp. 643-647, 2000.

[33] F. Glover and M. Laguna, “Tabu search,” in Modern Heuristic Techniques for Combinatorial Problems (C.R. Reeves, ed.), pp. 70-150, McGraw-Hill Book Company, 1995.

[34] N. Nicolici, "Power minimisation techniques for testing low power VLSI circuits," Tech. Rep. Upgrade from MPhil to PhD, University of Southampton, UK, December 1999.

[35] P. Kollig and B.M. Al-Hashimi, "A new approach to simultaneous scheduling, allocation and binding in high level synthesis," IEE Electronics Letters, vol. 33, pp. 1516-1518, Aug 1997.

[36] P. Kollig, Algorithms for Scheduling, Allocation and Binding in High Level Synthesis. PhD thesis, Staffordshire University, UK, Apr 1998.

[37] A. Majumdar and S.B.K. Vrudhula, "Fault coverage and test length estimation for random pattern testing," IEEE Transactions on Computers, vol. 44, pp. 234-247, Feb 1995. 
[38] AMS, 0.35 Micron CMOS Process Parameters. Austria Mikro Systeme International AG, 1998.

[39] H.K. Lee and D.S. Ha, "An efficient forward fault simulation algorithm based on the parallel pattern single fault propagation," in Proc. IEEE International Test Conference, pp. 946-955, 1991.

[40] S.P. Lin, A Design System to Support Built-In Self-Test of VLSI Circuits Using BILBOOriented Test Methodologies. PhD thesis, University of Southern California, May 1994. 


\section{List of Figures}

1 Comparison of data path testing using the traditional BIST embedding methodology and the proposed BIST methodology . . . . . . . . . . . . 27

2 Example of data path testing using the proposed TCC grouping methodology . 27

3 Tabu search-based testable design space exploration . . . . . . . . . . . 28

4 Reduction in test application time, BIST area overhead, number of test registers, volume of output data and overall test application time for complex hypothetical data paths using the TCC grouping methodology . . . . . . . . . . . . . 29

5 Comparison in fault-escape probability when 1 to 8 same-type modules are tested simultaneously in BIST embedding and TCC grouping methodologies . . 30 


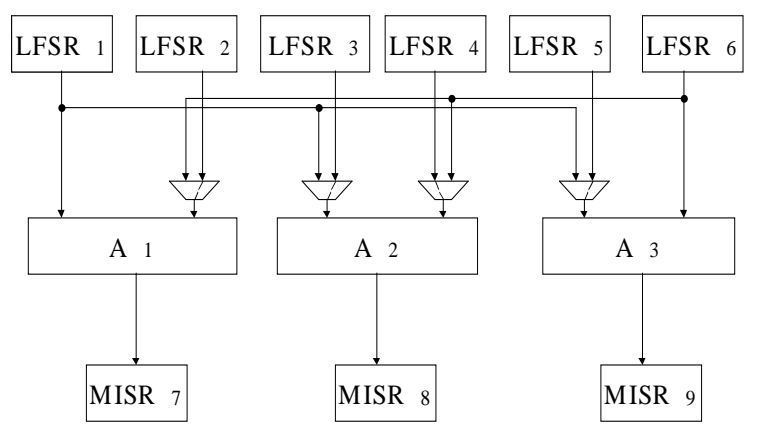

(a) BIST embedding methodology

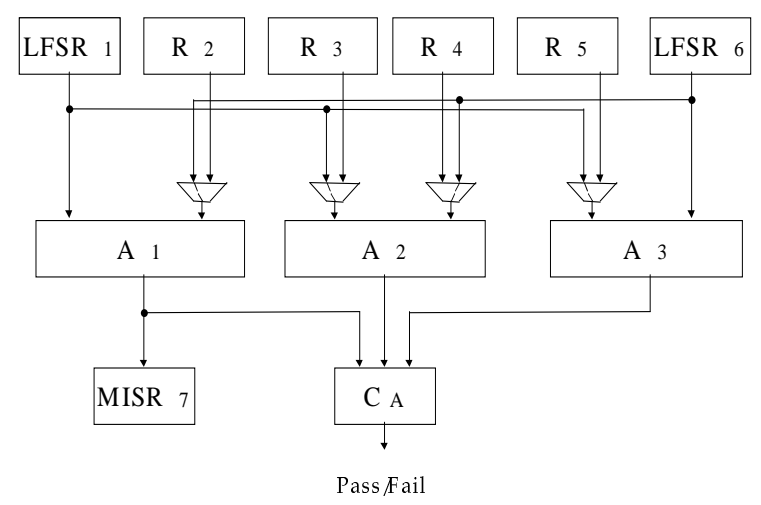

(b) The proposed BIST methodology

Figure 1: Comparison of data path testing using the traditional BIST embedding methodology and the proposed BIST methodology
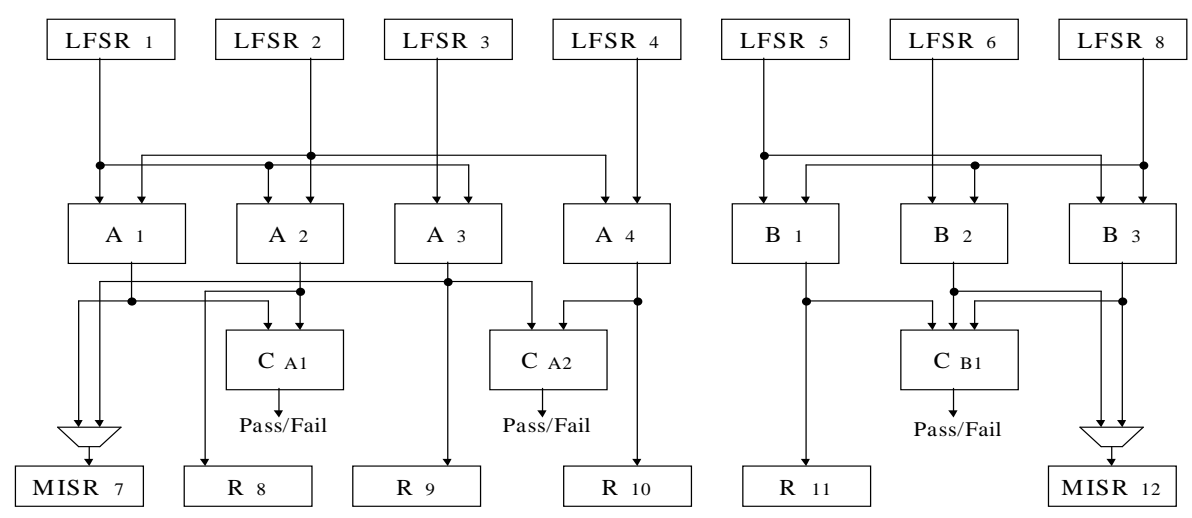

Figure 2: Example of data path testing using the proposed TCC grouping methodology 


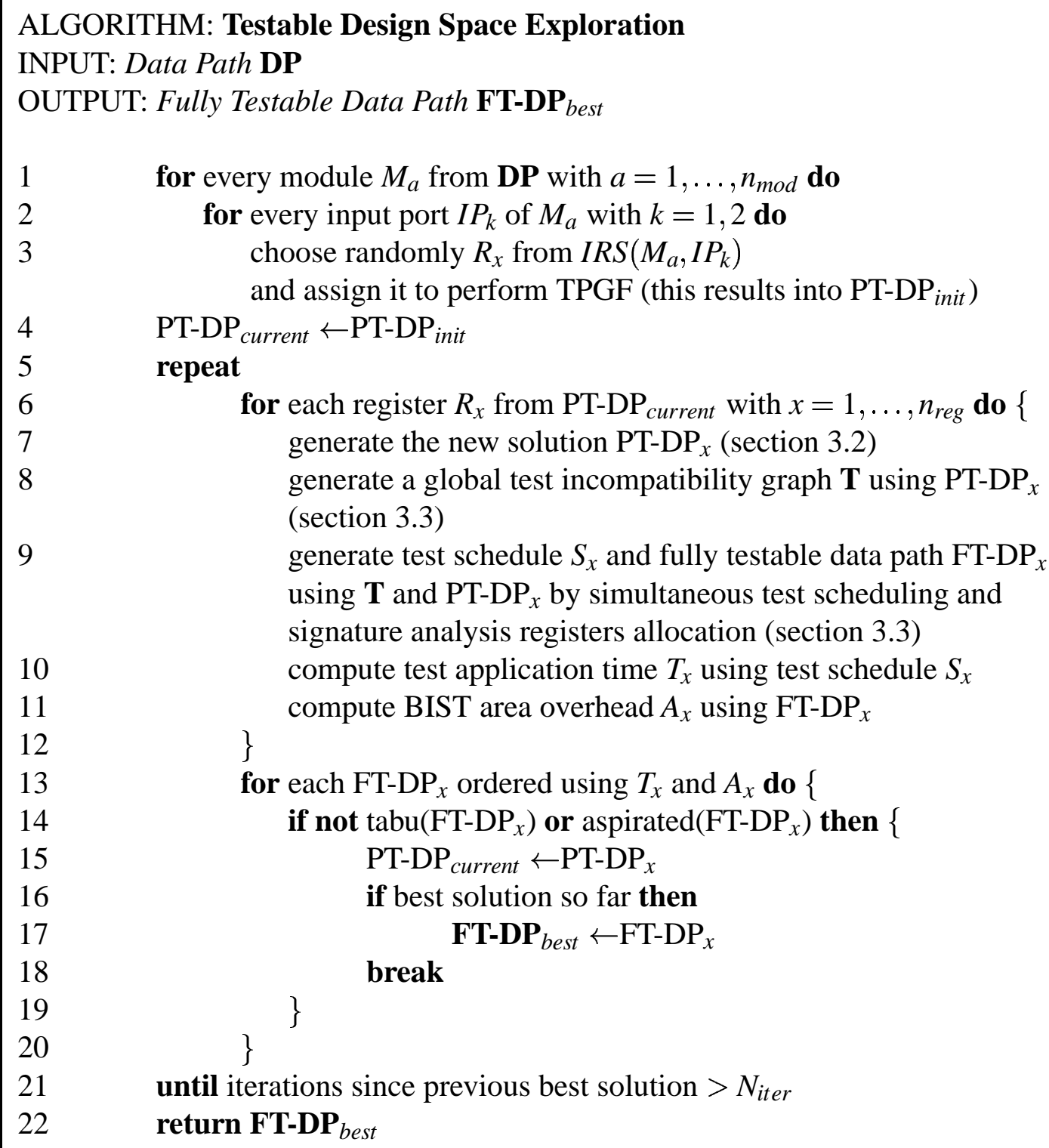

Figure 3: Tabu search-based testable design space exploration 


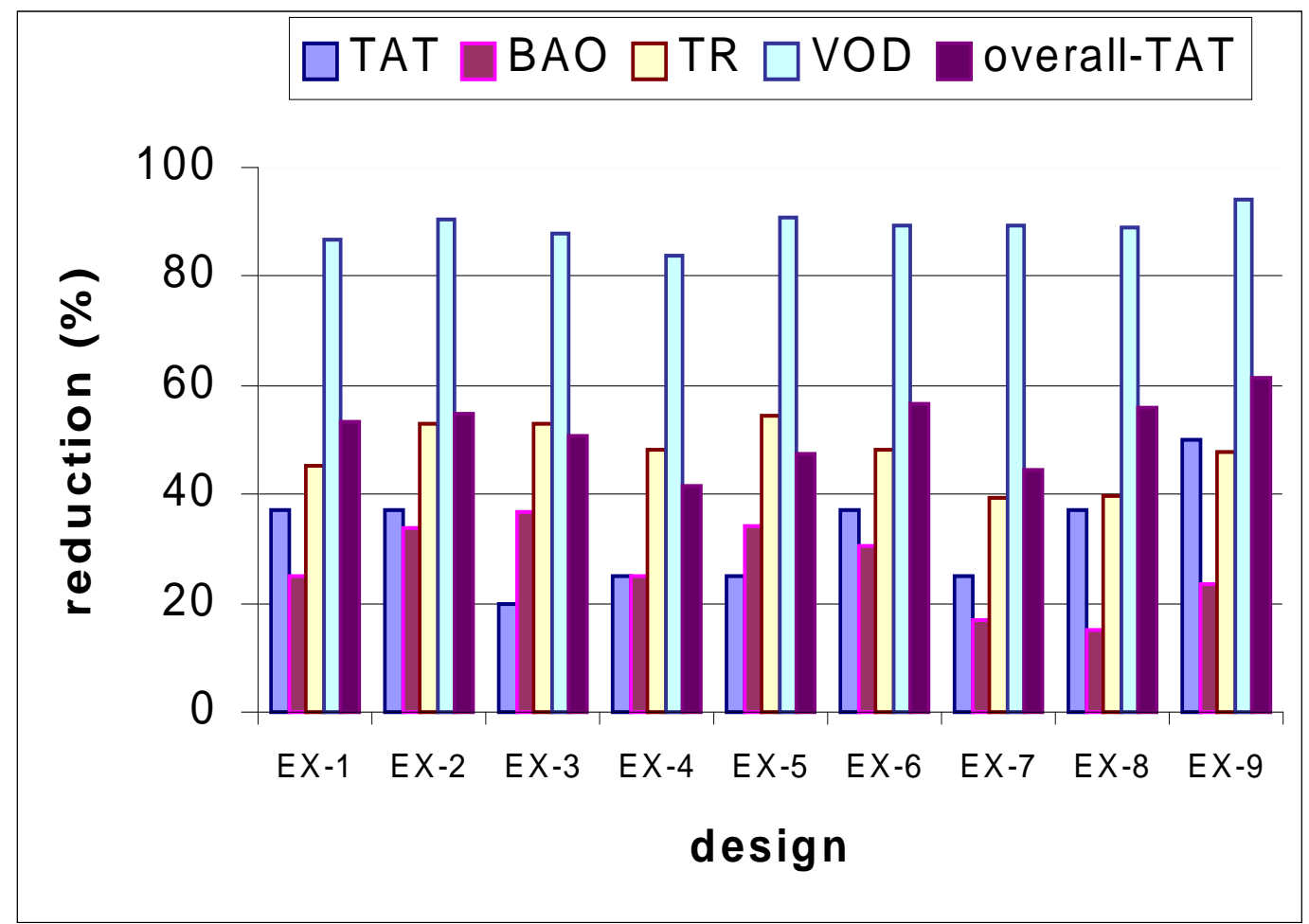

Figure 4: Reduction in test application time, BIST area overhead, number of test registers, volume of output data and overall test application time for complex hypothetical data paths using the TCC grouping methodology 


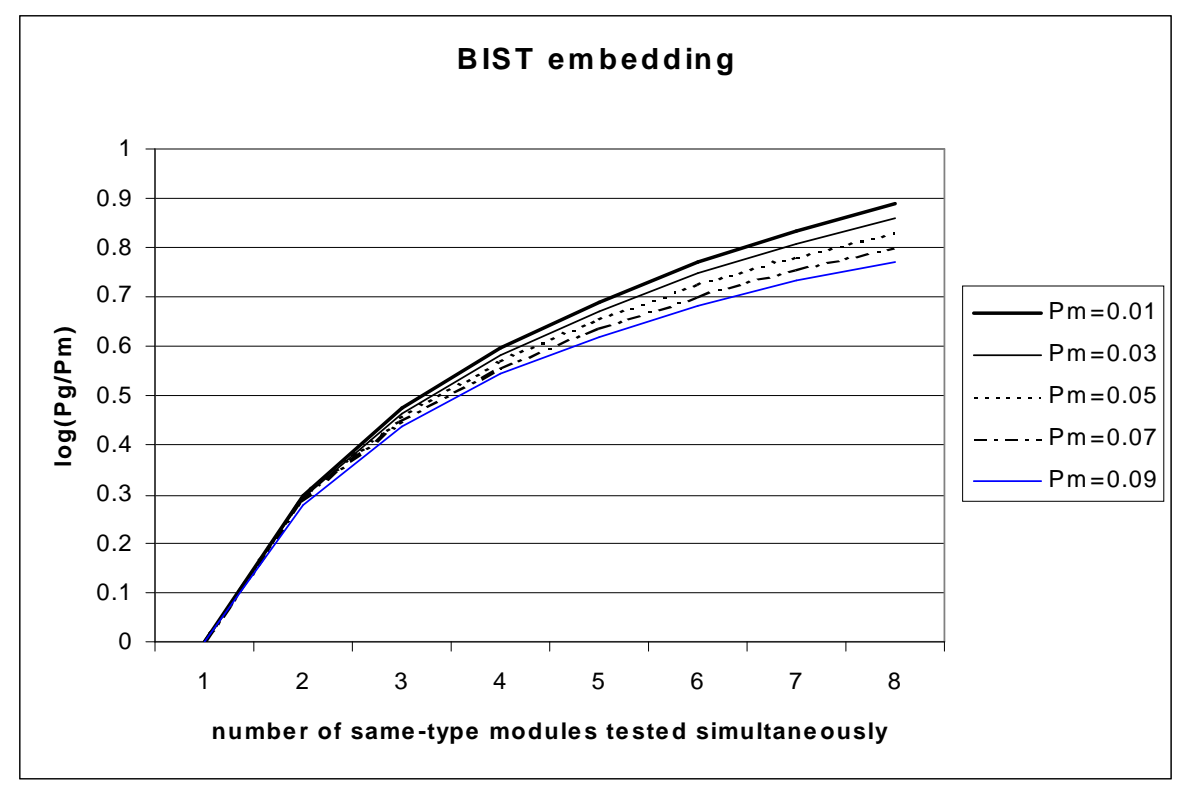

(a) Increase in fault-escape probability for BIST embedding

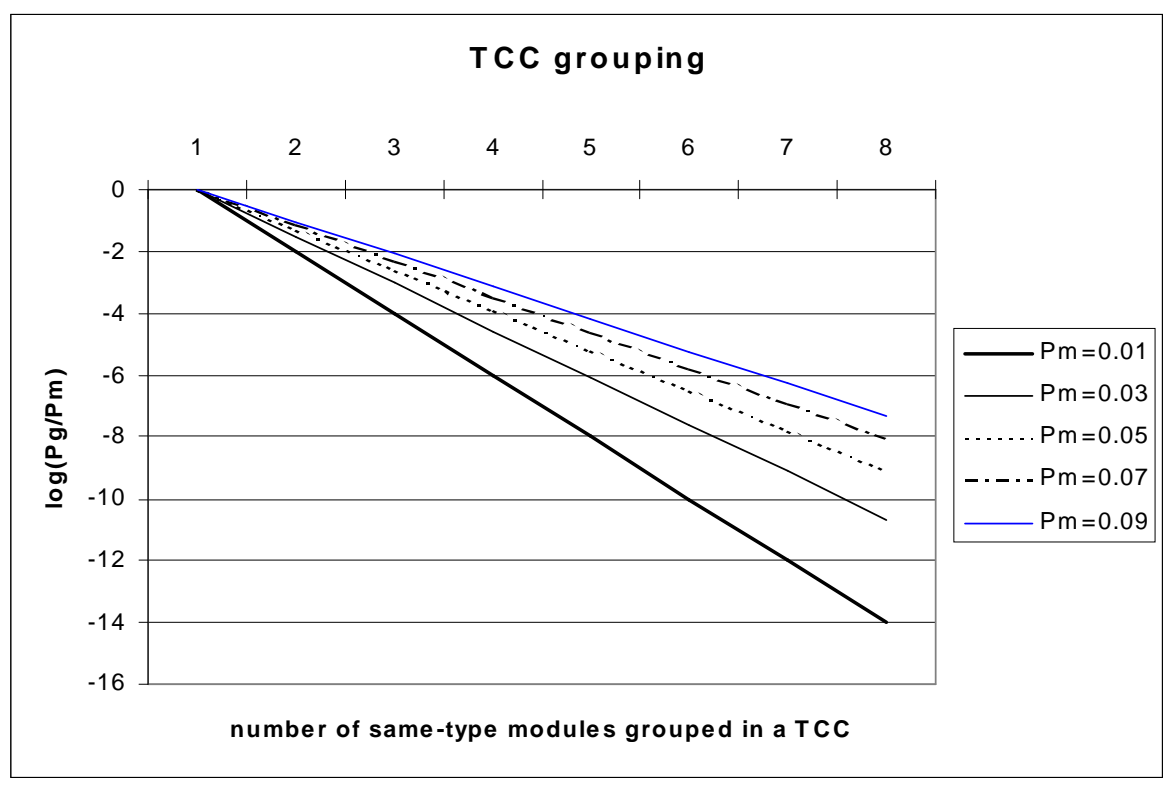

(b) Decrease in fault-escape probability for TCC grouping

Figure 5: Comparison in fault-escape probability when 1 to 8 same-type modules are tested simultaneously in BIST embedding and TCC grouping methodologies 


\section{List of Tables}

1 Comparison of BIST resources and test application time using the TCC grouping and the BIST embedding methodologies for benchmark examples . . . . . 32

2 Reduction in test application time, BIST area overhead, number of test registers, volume of output data and overall test application time for benchmark examples using the TCC grouping methodology . . . . . . . . . . . . . . . 33 


\begin{tabular}{|c|c|c|c|c|c|c|c|c|}
\hline \multirow{3}{*}{ Design } & \multirow{3}{*}{$\begin{array}{l}\text { M } \\
\mathbf{O} \\
\text { D }\end{array}$} & \multirow{3}{*}{$\begin{array}{l}\mathbf{R} \\
\mathrm{E} \\
\mathbf{G}\end{array}$} & \multicolumn{2}{|c|}{ BIST embedding } & \multicolumn{3}{|c|}{ TCC grouping } & \multirow{3}{*}{$\begin{array}{r}\text { CPU } \\
\text { time } \\
(\mathrm{s})\end{array}$} \\
\hline & & & \multirow{2}{*}{$\begin{array}{c}\text { BIST resources } \\
\text { Test registers }\end{array}$} & \multirow{2}{*}{ TAT } & \multicolumn{2}{|c|}{ BIST resources } & \multirow{2}{*}{ TAT } & \\
\hline & & & & & Test Registers & Comparators & & \\
\hline$E W F-17$ & $3 * 3+$ & 12 & 6 LFSR, 3 MISR & $5 \times T$ & 5 LFSR, 2 MISR & $2 \mathrm{C} 3$ & $4 \times T$ & 1.05 \\
\hline$E W F-18$ & $2^{*}, 3+$ & 12 & 5 LFSR, 4 MISR & $4 \times T$ & LFSR, 1 MISR & $\mathrm{C} 3,1 \mathrm{C} 2$ & $4 \times T$ & 2.17 \\
\hline$E W F-19$ & $2^{*}, 2+$ & 12 & FSR, 4 & $4 \times T$ & LFSR, 2 MISR & $2 \mathrm{C} 2$ & $4 \times T$ & 0.73 \\
\hline$E W F-20$ & $2^{*}, 2+$ & 12 & $\mathrm{R}$ & $4 \times T$ & MISR & 2 & $4 \times T$ & 0.75 \\
\hline$E W F-21$ & $2^{*}, 3+$ & 13 & $\mathrm{R}$ & $5 \times T$ & 2 MISR & $2 \mathrm{C} 2$ & $4 \times T$ & 1.58 \\
\hline$E W F-23$ & $1 *, 2+$ & 11 & $5 \mathrm{LF}$ & $4 \times T$ & 2 MISR & $1 \mathrm{C} 2$ & $4 \times T$ & 0.69 \\
\hline $8 D C T-10$ & $4 *, 4+$ & 15 & 7 LFSR, 6 & $5 \times T$ & 3 MISR & $2 \mathrm{C} 2,1 \mathrm{C} 4$ & $4 \times T$ & 2.56 \\
\hline $8 D C T-11$ & $4 *, 3+$ & 15 & 8 LFSR, 7 MISR & $5 \times T$ & 6 LFSR, 2 MISR & $1 \mathrm{C} 3,1 \mathrm{C} 4$ & $4 \times T$ & 2.57 \\
\hline $8 D C T-12$ & $4 *, 3+$ & 16 & 8 LFSR, 6 MISR & $5 \times T$ & 8 LFSR, 2 MISR & $1 \mathrm{C} 3,1 \mathrm{C} 4$ & $4 \times T$ & 1.86 \\
\hline $8 D C T-13$ & $4 *, 4+$ & 16 & 9 LFSR, 5 MISR & $5 \times T$ & 8 LFSR, 2 MISR & $2 \mathrm{C} 4$ & $4 \times T$ & 2.67 \\
\hline $8 D C T-14$ & $3 * 3+$ & 16 & 9 LFSR, 6 MISR & $4 \times T$ & 5 LFSR, 2 MISR & $2 \mathrm{C} 3$ & $4 \times T$ & 1.20 \\
\hline $8 D C T-16$ & $3 *, 2+$ & 16 & 7 LFSR, & $4 \times T$ & 5 LFSR, 2 MISR & $1 \mathrm{C} 2,1 \mathrm{C} 3$ & $4 \times T$ & 1.15 \\
\hline $32 D C T-30$ & $9 *, 12+$ & 60 & 33 LFSR, 21 MISR & $4 \times T$ & 18 LFSR, 2 MISR & $1 \mathrm{C} 5,1 \mathrm{C} 7,1 \mathrm{C} 9$ & $4 \times T$ & 129.70 \\
\hline $32 D C T-31$ & $9^{*}, 12+$ & 62 & 33 LFSR, 21 MISR & $4 \times T$ & 2 MISR & $1 \mathrm{C} 5,1 \mathrm{C} 7,1 \mathrm{C} 9$ & $4 \times T$ & 124.40 \\
\hline $32 D C T-32$ & $8^{*}, 12+$ & 62 & $32 \mathrm{LFS}$ & $4 \times T$ & 2 MISR & $1 \mathrm{C} 4,2 \mathrm{C} 8$ & $4 \times T$ & 103.70 \\
\hline $32 D C T-33$ & $8^{*}, 11+$ & 62 & $30 \mathrm{LF}$ & $4 \times T$ & 2 MISR & $1 \mathrm{C} 5,1 \mathrm{C} 6,1 \mathrm{C} 8$ & $4 \times T$ & 55.00 \\
\hline $32 D C T-37$ & $8^{*}, 9+$ & 63 & $26 \mathrm{LF}$ & $4 \times T$ & 2 MISR & $1 \mathrm{C} 3,1 \mathrm{C} 6,1 \mathrm{C} 8$ & $4 \times T$ & 86.64 \\
\hline $32 D C T-38$ & $9 *, 9+$ & 59 & $27 \mathrm{LFS}$ & $4 \times T$ & 2 MISR & $2 \mathrm{C} 9$ & $4 \times T$ & 38.47 \\
\hline $32 D C T-39$ & $8 *, 9+$ & 60 & $26 \mathrm{LFS}$ & $4 \times T$ & 2 MISR & $1 \mathrm{C} 8,1 \mathrm{C} 9$ & $4 \times T$ & 45.46 \\
\hline $32 D C T-40$ & $7 *, 10+$ & 61 & 27 LFSR, 17 MISR & $4 \times T$ & 16 LFSR, 2 MISR & $1 \mathrm{C} 7,1 \mathrm{C} 10$ & $4 \times T$ & 45.24 \\
\hline
\end{tabular}

Table 1: Comparison of BIST resources and test application time using the TCC grouping and the BIST embedding methodologies for benchmark examples 


\begin{tabular}{|c|c|c|c|c|c|c|c|}
\hline \multirow{3}{*}{ Design } & \multirow{3}{*}{$\begin{array}{c}\text { TAT } \\
\text { reduction } \\
(\%)\end{array}$} & \multirow{2}{*}{\multicolumn{3}{|c|}{$\begin{array}{c}\begin{array}{c}\text { BIST area overhead } \\
\text { reduction }(\%)\end{array} \\
\text { Data path width }\end{array}$}} & \multirow{3}{*}{$\begin{array}{c}\text { TR } \\
\text { reduction } \\
(\%)\end{array}$} & \multirow{3}{*}{$\begin{array}{l}\text { VOD } \\
\text { reduction } \\
(\%)\end{array}$} & \multirow{3}{*}{$\begin{array}{c}\text { overall- } \\
\text { TAT } \\
\text { reduction } \\
(\%)\end{array}$} \\
\hline & & & & & & & \\
\hline & & 4 bit & 8 bit & 16 bit & & & \\
\hline$E W F-17$ & 20 & $\begin{array}{l}-14.29 \\
\end{array}$ & -17.95 & -20.00 & 22.22 & 33.33 & 20.93 \\
\hline$E W F-18$ & 0 & 3.17 & 0.00 & -1.78 & 33.33 & 75.00 & 8.33 \\
\hline$E W F-19$ & 0 & 18.57 & 16.15 & 14.80 & 40.00 & 50.00 & 5.56 \\
\hline$E W F-20$ & 0 & 18.57 & 16.15 & 14.80 & 40.00 & 50.00 & 5.56 \\
\hline$E W F-21$ & 20 & -1.59 & -4.27 & -5.78 & 22.22 & 50.00 & 22.73 \\
\hline$E W F-23$ & 0 & 11.61 & 10.10 & 9.25 & 25.00 & 33.33 & 2.86 \\
\hline $8 D C T-10$ & 20 & -18.13 & -21.60 & -23.54 & 15.38 & 50.00 & 23.91 \\
\hline $8 D C T-11$ & 20 & 20.95 & 18.46 & 17.07 & 46.67 & 71.43 & 27.66 \\
\hline $8 D C T-12$ & 20 & 1.02 & -1.65 & -3.14 & 28.57 & 66.67 & 26.09 \\
\hline $8 D C T-13$ & 20 & -3.06 & -6.04 & -7.71 & 28.57 & 60.00 & 24.44 \\
\hline $8 D C T-14$ & 0 & 31.43 & 29.23 & 28.00 & 53.33 & 66.67 & 10.53 \\
\hline $8 D C T-16$ & 0 & 19.05 & 16.67 & 15.33 & 41.67 & 60.00 & 8.11 \\
\hline $32 D C T-30$ & 0 & 41.14 & 39.25 & 38.19 & 62.96 & 90.48 & 35.85 \\
\hline $32 D C T-31$ & 0 & 39.29 & 37.39 & 36.33 & 61.11 & 90.48 & 35.85 \\
\hline $32 D C T-32$ & 0 & 43.82 & 41.94 & 40.88 & 65.38 & 90.00 & 34.62 \\
\hline $32 D C T-33$ & 0 & 45.63 & 43.72 & 42.65 & 67.35 & 89.47 & 33.33 \\
\hline $32 D C T-37$ & 0 & 33.72 & 31.75 & 30.65 & 55.81 & 82.35 & 28.57 \\
\hline $32 D C T-38$ & 0 & 37.46 & 35.56 & 34.49 & 60.00 & 88.89 & 32.00 \\
\hline $32 D C T-39$ & 0 & 33.55 & 31.66 & 30.60 & 55.81 & 88.24 & 30.61 \\
\hline $32 D C T-40$ & 0 & 37.34 & 35.49 & 34.45 & 59.09 & 88.24 & 30.61 \\
\hline
\end{tabular}

Table 2: Reduction in test application time, BIST area overhead, number of test registers, volume of output data and overall test application time for benchmark examples using the TCC grouping methodology 